\title{
An Optimal Transportation Schedule of Mobile Equipment
}

\author{
S. Guillén-Burguete ${ }^{1}$, H. Sánchez-Larios ${ }^{*^{2}}$, J.G Vázquez-Vázquez ${ }^{3}$ \\ ${ }^{1}$ Instituto de Ingeniería, Universidad Nacional Autónoma de México \\ México, D.F., México \\ ${ }^{2}$ Facultad de Ingeniería, Universidad Nacional Autónoma de México \\ México, D. F., México \\ *herica.sanchez@ciencias.unam.mx \\ ${ }^{3}$ Facultad de Ciencias, Universidad Nacional Autónoma de México \\ México, D.F., México
}

\begin{abstract}
Motivated by a problem faced by road construction companies, we develop a new model to obtain an optimal transportation schedule of mobile machines which have to travel to execute tasks. In this problem, each task is characterized by the location where it is to be executed, a work-content in terms of machine-time units, and one or more time intervals within which it can be performed. The machines can be transported from one location to another at any time, thus the problem has an indefinite number of variables. However, this indefinite number of variables can be reduced to a definite one because, as we prove, the problem has an optimal solution in which the arrivals of machines occur only at certain time instants. The objective is to minimize the total transportation cost such that all the tasks are executed within their time intervals. The constraints ensuring that the tasks are processed within their prescribed time intervals are nonlinear; nevertheless, due to the sets of the possible arrival times of the machines forming bounded convex polyhedra, our problem can be transformed into a mixed integer linear program by the same device used in the decomposition principle of Dantzig-Wolfe.
\end{abstract}

Keywords: transportation schedule, generalized linear programming, bounded convex polyhedron, work-content.

\section{RESUMEN}

Motivados por un problema que enfrentan las compañías de la construcción, desarrollamos un modelo nuevo para obtener un calendario óptimo del transporte de máquinas móviles que tienen que viajar para realizar tareas. En este problema, cada tarea está caracterizada por el lugar donde ésta tiene que ser realizada, una carga de trabajo en términos de tiempo-máquina, y uno o más intervalos de tiempo dentro de los cuales la tarea puede ser procesada. Las máquinas se pueden transportar desde un lugar hasta otro en cualquier momento, por lo tanto el problema tiene un número indefinido de variables. Sin embargo, este número indefinido de variables se puede reducir a uno definido porque, como se demuestra, el problema tiene una solución óptima en la que las llegadas de las máquinas ocurren solamente en ciertos momentos. El objetivo es minimizar el costo total de transporte tal que todas las tareas sean ejecutadas dentro de sus intervalos de tiempo. Las restricciones que aseguran que cada tarea sea procesada dentro de sus intervalos de tiempo prescritos son no lineales; sin embargo, debido a que los conjuntos de los posibles tiempos de llegada de las máquinas forman poliedros convexos acotados, nuestro problema puede transformarse en un programa lineal entero mixto por el mismo artificio usado en el principio de descomposición de Dantzig-Wolfe.

\section{Introduction}

In this paper, we develop a new model to obtain an optimal transportation schedule of identical mobile machines which have to execute tasks located at several places. Our work is motivated by a transportation scheduling problem faced by road construction and maintenance companies. In this problem, each task is characterized by the location where it is to be executed, a work-content in terms

of machine-time units (e.g., machine-hours), and one or more work intervals (time intervals within which it is possible to process the work-content of a task). In practice, these work intervals are determined according to external factors such as the weather, or constructions contracts. We consider the following assumptions. At each location, there may be more than one task and the 
work intervals associated with those tasks do not overlap. The tasks can be started at any time within their respective work intervals and executed with interruptions. Moreover, it is considered that the machines can be transported from one location to another location at any time and that they are busy at all work-instant (a time instant within a work interval) unless the corresponding task has been completed. Finally, we consider that the work-content of a task may be processed by several machines simultaneously without altering the processing speed of each machine. The transportation times and the transportation costs are given. Also given is the number of machines available at each location at the beginning of the planning period. The objective is to minimize the total transportation cost in such a way that all the tasks are accomplished within their respective prescribed work intervals. This problem is NP-hard as it is a generalization of the traveling salesman problem.

The proposed model can be applied, for instance, when machines performing large tasks in different places have to travel between tasks. In this case, "machines" may represent harvesters, dirt movers, excavators, land levelers, or asphalt mixers, and "tasks" may be activities in agriculture, forestry, construction and maintenance projects. Also, our model can be applied in manufacturing systems where machines travel instead of tasks. This occurs, for example, when the products to be manufactured are too big or heavy (e.g., ships) to be moved between machines.

Achieving high equipment utilization is an important concern among industries due to the large capital investments on machines and equipment. In this sense, our model can be applied in situations where economic resources are so limited that the optimal use of expensive machines implies that the machines must be shared among the locations where the tasks are to be performed; under these conditions, the purchase of machines is convenient only if the number of available machines is insufficient for performing the tasks.

As far as we know, the problem that we formulate in this paper has not been addressed in the literature. Because the machines travel between the tasks, one could think that the proposed problem is of the type of routing flow shop (see, e.g., Averbakh and Berman, 1999), routing open shop (see, e.g., Averbakh et al., 2006) or routingscheduling problems (see e.g., Bredström and Rönnqvist, 2008; Kek et al., 2008; Kim et al., 2006; Desaulniers et al., 1998; Desrosiers et al., 1995). However, our problem cannot be obtained from these models because of some of its mentioned characteristics: the tasks can be executed with interruptions and are specified by a work-content in terms of machine-time units instead of a fixed duration and fixed machine requirements, and each machine may revisit any location any number of times.

We develop the formulation of the problem in three stages. Each stage consists of a problem simpler than the previous one with an optimal solution which is also an optimal solution of the previous problem. Each of the three problems has two sets of constraints. The constraints of the first set are restrictions on the availability of machines at each location. The constraints of the second set ensure that each task is accomplished within its prescribed work intervals; each of these constraints has products of two quantities: the number of machines arriving at a location during a work interval and the amount of time that these machines work during that work interval. In the first two stages of the formulation, these two quantities are decision variables, and hence the first two problems are nonlinear.

The paper is organized as follows: In Section 2, we develop the preliminary stage (Problem 1) in which it is considered that the machines can be transported from one location to another location at any time. Thus, Problem 1 has an indefinite number of variables. In Section 3, we formulate Problem 2 (second stage of the formulation of our model) which is obtained from Problem 1 by reducing the set of time instants at which it is permissible to transport machines. This reduction is based on the fact (that we prove in Theorem 1) that Problem 1 has an optimal solution in which the arrival time of any shipment of machines (one or more machines are transported together at the same time) is a work-instant. In this optimal solution, each shipment of machines belongs to one of the following two classes of shipments. A class $A$ shipment: one or more machines depart together from a location $L$ at some idle-instant (a time instant outside of a work interval) and arrive together at another location $M$ just at the beginning of a work interval. A class $B$ shipment: one or more 
machines depart together from a location $L$ at a work-instant within a work interval $i$ and arrive together at another location $M$ at a work-instant within a work interval $j$. We prove (Theorem 2) that an arbitrary number of class $B$ shipments, each of them departing from location $L$ at an instant within work interval $i$ and arriving at location $M$ at an instant within work interval $j$, is equivalent, in the sense of the portions of work-content processed by the machines transported in these shipments, to a single consolidated shipment (several shipments departing and arriving together) departing from $L$ at certain work-instant within $i$ and arriving at $M$ at a work-instant within $j$. Theorem 2 allow us to formulate Problem 2 with a definite number of variables. However, this problem is still nonlinear and cannot be solved directly because its constraints on the machines availability at each location in the interior of the work intervals cannot be expressed in terms of the decision variables. We prove that it is possible to find an optimal solution of Problem 2 through an iterative procedure in which the relaxed Problem 2 (Problem 2'), obtained by dropping the constraints on the machines availability, is solved iteratively by dividing, according to certain strategy, the work intervals into adjacent subintervals until the solution satisfies such constraints. In Section 4, we develop the third stage of the formulation of our model. In this section, we will see that Problem 2' can be considered as a generalized linear program and that it can be transformed into a mixed integer linear program (Problem 3) by the same device used in the decomposition principle of DantzigWolfe. More precisely, we show (Theorem 3 ) that any single consolidated class $B$ shipment is equivalent (in the above-mentioned sense) to a pair of "extreme" shipments. This equivalence is because the set of all the possible arrival times of each class $B$ shipment is a bounded convex polyhedron. Finally, in Section 5, some conclusions are given.

\section{Problem 1: A nonlinear program with an indefinite number of variables}

Let $K=\{1, \ldots, k, \ldots, q\}$ denote the set of tasks to be executed, and let $\Lambda=\{1, \ldots, l, \ldots, m\}$ denote the set of locations where either the tasks are to be executed or there are machines. The location where the task $k \in K$ is to be performed is given by a function $\lambda^{\prime}: K \rightarrow \Lambda$. Let $I=\{1, \ldots, i, \ldots, n\}$ denote the set of all the work intervals, and let $I_{k}$ denote the set of the work intervals associated with the task $k \in K$; obviously, $I=\cup_{k \in K} I_{k}$, and $I_{k} \cap I_{h}=\varnothing$ for all $k$, $h \in K$ with $k \neq h$. We define the function $\lambda: I \rightarrow \Lambda$ as $\lambda(i)=p$ if, and only if, $i \in I_{k}$ and $\lambda^{\prime}(k)=p$. That is to say, $\lambda(i)$ is the location corresponding to the work interval $i \in l$. Thus, if $i \in I_{k}$ then $\lambda(i)=\lambda^{\prime}(k)$. For simplicity reasons, it is assumed that at each location the work intervals do not overlap. Each task $k \in K$ has associated with it, in addition to a set of work intervals $I_{k}$ and a location $\lambda^{\prime}(k)$, a workcontent $w_{k}$ which must be processed within $I_{k}$. The beginning $E_{i}$ and the end $L_{i}\left(E_{i}<L_{i}\right)$ of each work interval $i \in I$ are known. We denote by $\tau(i)$ each work interval $\left[E_{i}, L_{i}\right), i \in l$, and by $T$ the planning period given by $T:=\left[\inf \left\{E_{i}-\sup \{T(p, \lambda(i)) \mid p \in \Lambda\} \mid\right.\right.$ $\left.i \in I\}, \sup \left\{L_{i} \mid i \in I\right\}\right]$. The number of machines $Q_{p}$ available at $p \in \Lambda$ at the beginning of the planning period is given. Also given are the cost of transporting a machine from one location $p$ to another location $s, C(p, s)$, and the corresponding transportation time, $T(p, s)$.

In order to guarantee the existence of a feasible solution, we assume that there are one or more dummy locations in $\Lambda$ with an unlimited number of machines. These locations can be thought of as "stores of machines". If $p$ is a dummy location, then the transportation cost $C(p, s)$ includes the cost of acquiring a machine at the location $p$. The problem consists of finding a transportation schedule of machines in such a way that all the tasks are accomplished within their respective work intervals at minimum total transportation cost.

\subsection{Formulation of Problem 1}

In Problem 1, we consider that the machines can be transported from one location to another location at any time $t \in T$. The decision variables are the number of machines $u(p, s, t)$ transported from location $p \in \Lambda$ to location $s \in \Lambda$ with a departure time $t \in T$. If $u(p, s, t) \geq 1$; then these $u$ machines are transported together in one shipment departing from $p$ at time $t \in T$ and arriving at $s$ at time $t+T(p, s)$. 
The work-content processed during a work interval $\left[E_{i}, L_{i}\right), i \in l$, per machine that arrives at location $\lambda(i)$ at time $t \in T$ is denoted by $G(i, t)$ and is given by

$$
\begin{aligned}
& G(i, t):=\left\{\begin{array}{cc}
L_{i}-E_{i} & \text { if } t<E_{i} \\
L_{i}-t & \text { if } E_{i} \leq t<L_{i} \\
0 & \text { if } L_{i} \leq t
\end{array}\right\}= \\
& =\max _{i \in I}\left\{0,\left(L_{i}-\max \left\{t, E_{i}\right)\right\}, \quad i \in I .\right.
\end{aligned}
$$

Equivalently, $G(i, t)$ is the work-content without processing during work interval $i \in I$ per machine that leaves location $\lambda(i)$ at time $t \in T$.

Problem 1 is formulated as follows, where the set of times $t \in T$ such that $u(p, s, t)>0$ is denoted by $\Gamma($ $p, s)$ :

$$
\text { Minimize } \sum_{t \in \Gamma(p, s)} \sum_{p, s \in \Lambda} C(p, s) u(p, s, t)
$$

Subject to

$$
\begin{aligned}
& \sum_{\substack{t^{\prime} \in \Gamma(p, s), t^{\prime}<t}} \sum_{s \in \Lambda} u\left(p, s, t^{\prime}\right)- \\
& -\sum_{t^{\prime}-T(s, p) \in \Gamma(s, p),} \sum_{\substack{t^{\prime}<t \\
p \in \Lambda}} u\left(s, p, t^{\prime}-T(s, p)\right) \leq Q_{p}, \\
& \sum_{i \in I_{k}}\left[-\sum_{\substack{t^{\prime} \in \Gamma(\lambda(i), s), t^{\prime}<L_{i}}} \sum_{s \in \Lambda} G\left(i, t^{\prime}\right) u\left(\lambda(i), s, t^{\prime}\right)+\right. \\
& +\sum_{t^{\prime}-T(s, \lambda(i)) \in \Gamma(s, \lambda(i)),} \sum_{t^{\prime}<L_{i}} G \in \Lambda \\
& \geq w_{k}-Q_{\lambda^{\prime}(k)} \sum_{i \in l_{k}}\left(L_{i}-E_{i}\right), \forall k \in K ; \\
& u(p, s, t) \geq 0, \quad p, s \in \Lambda, \text { for all } t \in T ;
\end{aligned}
$$

$$
u(p, s, t) \text { integer, } p, s \in \Lambda \text {, for all } t \in T \text {. }
$$

The objective function (2) minimizes the total transportation cost. Constraints (3) represent the restrictions on the machines availability at each location at all instant $t \in T$. Constraints (4) state that each task $k \in K$ must be accomplished within its work intervals $i \in I_{k}$. Finally, constraints (5) and (6) impose, respectively, nonnegativity and integrality conditions on the variables $u(p, s, t)$.

As one can see, constraints (4) are nonlinear. Each term of the first triple sum of the left-hand side of Equation 4 is the portion of work-content $G\left(i, t^{\prime}\right) u\left(\lambda(i), s, t^{\prime}\right)$ of the task $k$ that $u\left(\lambda(i), s, t^{\prime}\right)$ machines, for departing from location $\lambda(i)$ at time $t^{\prime}$ $<L_{i}$, stop processing at $\lambda(i)$ during the lapse of time $\left[\max \left(t^{\prime}, E_{i}\right), L_{i}\right) \subseteq\left[E_{i}, L_{i}\right), i \in I_{k}$; each term of the second triple sum of the left-hand side of Equation 4 is the portion of work-content $G\left(i, t^{\prime}\right) u(s, \lambda(i)$, $\left.t^{\prime}-T(s, \lambda(i))\right)$ of the task $k$ that $u\left(s, \lambda(i), t^{\prime}-T(s, \lambda(i))\right)$ machines, after arriving at location $\lambda(i)$ at time $t^{\prime}<$ $L_{i}$, process during the lapse of time $\left[\max \left(t^{\prime}, E_{i}\right), L_{i}\right)$ $\subseteq\left[E_{i}, L_{i}\right), i \in I_{k}$; the right-hand side of Equation 4 is the portion of work-content of the task $k$ that is not processed by the $Q_{\lambda^{\prime}(k)}$ machines initially located at location $\lambda^{\prime}(k)$. Problem 1 is a nonlinear program with an indefinite number of variables.

\section{Problem 2: A nonlinear program with a definite number of variables}

In this section, we prove the following statements which allow us to transform Problem 1 into a problem with a definite number of variables:

(1). Problem 1 has an optimal solution in which the arrival time of each shipment is a work-instant;

(2). Several class $B$ shipments, each of them departing from location $\lambda(i)$ at a work-instant within work interval $i$ and arriving at location $\lambda(j)$ at a work-instant within work interval $j$, can be replaced by a single consolidated shipment.

With regard to statement (1), the following theorem establishes that there is an optimal solution of Problem $1,\left\{u^{*}(\cdot, \cdot, \cdot)\right\}$, such that each shipment of $u^{*}(p, s, t)>0$ machines is either: (a) a class $A$ shipment, i.e., the departure time $t$ of the shipment $u^{*}(p, s, t)$ is an idle-instant and the arrival time $t+$ $T(p, s)$ of $u^{*}(p, s, t)$ is the beginning of a work interval at the arrival location $s$; or (b) a class $B$ shipment, i.e., the departure time $t$ of the shipment $u^{*}(p, s, t)$ is a work-instant and the arrival time $t+$ 
$T(p, s)$ of $u^{*}(p, s, t)$ is a work-instant at the arrival location $s$.

Theorem 1. There is an optimal solution of Problem 1, $\left\{u^{*}(\cdot, \cdot, \cdot)\right\}$, such that if $u^{*}(p, s, t)>0, p$ $\neq s$, then the following two conditions are satisfied:

(a) if $t \notin \tau(i), i \in I$ with $\lambda(i)=p$, then $t+T(p, s)=E_{j}$ for some $j \in I$ with $\lambda(j)=s$;

(b) if $t \in \tau(i) i \in I$ with $\lambda(i)=p$, then $t+T(p, s) \in \tau(j)$ for some $j \in I$ with $\lambda(j)=s$.

\section{Proof}

Due to the existence of at least one "store of machines", Problem 1 has an optimal solution $S$. Suppose that $u$ is a shipment belonging to $S$, then it is sufficient to prove that conditions (a) and (b) guarantee a local optimum:

Suppose that the shipment $u$ departs from location $p$ at some idle-instant. Then its arrival time at location $s$ at the beginning of a work interval is a local optimum because if the shipment arrived one delta of time earlier than the beginning of a work interval, then the sent machines would be idle that delta of time at the arrival location, and if this shipment arrived one delta of time later than the beginning of that work interval, then the sent machines would be idle for that delta of time at the departure location instead of working that delta of time at the arrival location.

Now suppose that the shipment $u$ departs from location $p$ at a work-instant $t$ within $\tau(i)$. If the arrival time of the shipment is an idle-instant, then the portion of work-content processed by the machines transported in this shipment could increase if the departure time of the shipment is delayed one delta of time; this possible increment is because the machines could be working that delta of time at the departure location instead of being idle at the arrival location. Therefore, the shipment $u$ can be replaced by another shipment $u^{*}$ departing from $p$ and that arrives at $s$ at the beginning $E_{j}$ of the work interval that is immediately after $t+T(p, s)$. If such $E_{j}$ does not exist, the shipment $u$ can be eliminated and in this case, the value of the objective function in $S$ does not increase. If $E_{j}$ exists, the departure time of $u^{*}$ is either a work-instant, in whose case $u^{*}$ satisfies condition (b), or an idle-instant, in whose case $u^{*}$ satisfies condition (a).

The set of all the possible departure times of the class $B$ shipments departing from location $\lambda(i)$ at a work-instant within the work interval $i$ and arriving at location $\lambda(j)$ at a work-instant within the work interval $j$ is the time interval $\tau^{\prime}(i, j):=\left[E_{i}, L_{i}\right) \cap\left[E_{j}-\right.$ $\left.T(\lambda(i), \lambda(j)), L_{j}-T(\lambda(i), \lambda(j))\right)$; the corresponding set of all the possible arrival times is the time interval $\tau(i, j):=\left[E_{i}+T(\lambda(i), \lambda(j)), L_{i}+T(\lambda(i), \lambda(j))\right) \cap$ $\left[E_{j}, L_{j}\right)$. We call $\tau(i, j)$ the projection of the work interval $\tau(i)$ onto the work interval $\tau(j)$.

In relation to statement (2), the following theorem establishes that if the projection of $\tau(i)$ onto $\tau(j), \tau(i$, $j$ ) is a non-empty set, then both the portion of workcontent processed by $Q$ machines at location $\lambda(i)$ during work interval $i$ and the portion of workcontent processed by these $Q$ machines at location $\lambda(j)$ during work interval $j$ remain unchanged if: a) the $Q$ machines are sent in several shipments from $\lambda(i)$ to $\lambda(j)$, where each shipment arrives at $\lambda(j)$ at an instant within the projection $\tau(i, j)$; or $b$ ), the $Q$ machines are sent together in a single consolidated shipment from $\lambda(i)$ to $\lambda(j)$, where the consolidated shipment of $Q$ machines departs from $\lambda(i)$ at a certain workinstant $t^{\prime} \in \tau^{\prime}(i, j)$ and arrives at $\lambda(j)$ at an instant within $\tau(i, j)$. The time $t^{\prime}$ is obtained in the same way as the application point of the resultant force of a system of parallel forces. Formally, we have the following theorem.

Theorem 2. Let $i, j \in I$ be two work intervals with $\lambda(i)$ $\neq \lambda(j)$ and $\tau(i, j) \neq \varnothing$, and let $U:=\left\{u\left(\lambda(i), \lambda(j), t_{h}\right) \mid\right.$ $u\left(\lambda(i), \lambda(j), t_{h}\right)>0, t_{h}+T(\lambda(i), \lambda(j)) \in \tau(i, j), i, j \in l$, and $h=1, \ldots, N\}$ be a set of $N$ shipments, each of them departing from $\lambda(i)$ and arriving at $\lambda(j)$ at an instant within the projection $\tau(i, j)$. Then, both the portion of work-content processed by the $\sum_{h} u\left(\lambda(i), \lambda(j), t_{h}\right)$ machines during work interval $i$ and the portion of work-content processed by the same machines during work interval $j$ remain unchanged if the $N$ shipments are replaced by a single consolidated shipment of

$u\left(\lambda(i), \lambda(j), t^{\prime}\right)=\sum_{h=1}^{N} u\left(\lambda(i), \lambda(j), t_{h}\right)$

machines, where the departure time $t^{\prime}$ is given by 


$$
t^{\prime}:=\frac{\sum_{h=1}^{N} t_{h} u\left(\lambda(i), \lambda(j), t_{h}\right)}{\sum_{h=1}^{N} u\left(\lambda(i), \lambda(j), t_{h}\right)} .
$$

Proof

Using Equations 1, 7 and 8, we can directly prove that the portion of work-content processed during work interval $j$ by the machines transported in the $N$ shipments, $u\left(\lambda(i), \lambda(j), t_{h}\right)$ where $h=1, \ldots, N$, is equal to the work-content processed during work interval $j$ by the machines $u\left(\lambda(i), \lambda(j), t^{\prime}\right)$ transported in the consolidated shipment:

$$
\begin{aligned}
\sum_{h=1}^{N} G\left(j, t_{h}+T(\lambda(i), \lambda(j))\right) u\left(\lambda(i), \lambda(j), t_{h}\right) \\
=\sum_{h=1}^{N}\left[L_{j}-t_{h}-T(\lambda(i), \lambda(j))\right] u\left(\lambda(i), \lambda(j), t_{h}\right) \\
=\left[L_{j}-t^{\prime}-T(\lambda(i), \lambda(j))\right] u\left(\lambda(i), \lambda(j), t^{\prime}\right) \\
=G\left(j, t^{\prime}+T(\lambda(i), \lambda(j))\right) u\left(\lambda(i), \lambda(j), t^{\prime}\right) .
\end{aligned}
$$

The proof is similar for the work interval $i$.

Notice that $t^{\prime}$ is a convex combination of $\left\{t_{1}, \ldots, t_{N}\right\}$, and then $t^{\prime}+T(\lambda(i), \lambda(j)) \in \tau(i, j)$. In the context of Theorem 2, the consolidated shipment of $u(\lambda(i)$, $\left.\lambda(j), t^{\prime}\right)$ machines is called the composition of the set $U$ of $N$ shipments, and the set $U$ of $N$ shipments is called a decomposition of $u(\lambda(i), \lambda(j)$, $\left.t^{\prime}\right)$ machines. Figure 1 depicts the projection $\tau(i, j)$ of a work interval $i$ onto a work interval $j$, the set $U$ of $N$ shipments, each of them departing from location $\lambda(i)=p$ at a time $t_{h} \in \tau^{\prime}(i, j)$ and arriving at location $\lambda(j)=s$ at a time $t_{h}+T(p, s) \in \tau(i, j)$, and the corresponding consolidated shipment which departs from location $\lambda(i)$ at $t^{\prime} \in \tau(i)$.

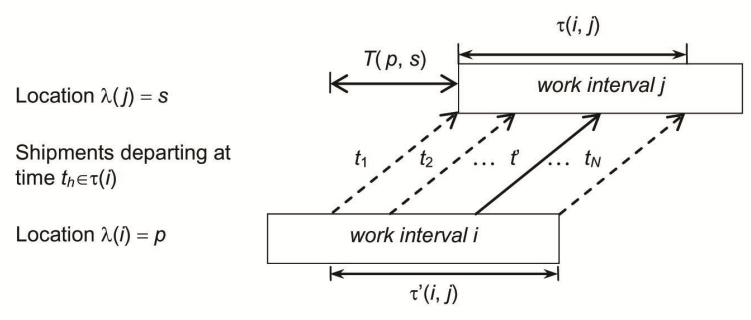

Figure 1. Composition and decomposition of shipments.
By Theorem 2, for each projection of a work interval onto another work interval, the indefinite number of class $B$ shipments can be replaced by a single consolidated shipment. Then by Theorem 1 and 2, we can formulate a new problem equivalent to Problem 1, which has a definite number of variables.

\subsection{Formulation of Problem 2}

In order to reformulate Problem 1 in terms of a definite number of variables (Problem 2), we use the fact that there exists an optimal solution of Problem 1 in which only class $A$ and $B$ shipments are involved in the transportation of machines and that there is only one class $B$ shipment for each projection $\tau(i, j)$. For this reformulation, we extend the notation used in Problem 1, as explained in (i) and (ii), and modify the formulation of constraints (3) according to the new decision variables, as pointed out in conditions (iii) and (iv):

(i) Class A shipments: $Z_{p j}:=u\left(p, s, E_{j}-T(p, s)\right)$ for each $j \in I$ and $p, s \in \Lambda$, such that $\lambda(j)=s \neq p$, and $E_{j}-$ $T(p, s) \notin \tau(i)$ for all $i \in I$ such that $\lambda(i)=p$.

(ii) Class $B$ shipments: $Y_{i j}:=u\left(p, s, t_{i j}\right)$ for each $i$, $j \in l$ and $p, s \in \Lambda$, such that $t_{i j}+T(p, s) \in \tau(i, j) \neq \varnothing$, and $\lambda(i)=p \neq s=\lambda(j)$.

(iii) Machine availability condition at the extremes of the work intervals: for each $i \in I$ constraint (3) is satisfied for the time instants $t=E_{i}$ and $t=L_{i}$.

(iv) Machine availability condition in the interior of the work intervals: for each $i \in I$ constraint (3) is satisfied for all instant $t=t_{i j} \in \tau^{\prime}(i, j) \neq \varnothing, j \in l$.

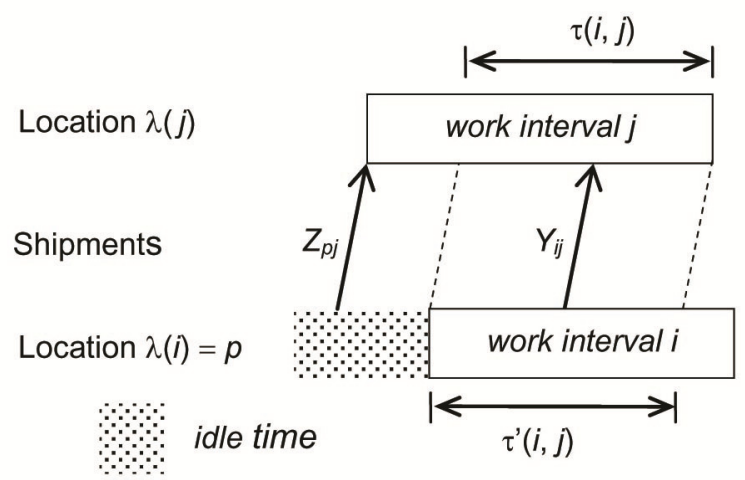

Figure 2. Class $A$ and $B$ shipments. 
Figure 2 illustrates a class $A\left(Z_{p j}\right)$ shipment and a class $B\left(Y_{i j}\right)$ shipment. In order to formalize Problem 2, we define the following sets: For each $i \in I$, a shipment $Z_{\lambda(i) j}$ that departs from $\lambda(i)$ at some idle-instant before $E_{i}$ arrives at $\lambda(j)$ at the beginning $E_{j}$ of a work interval $j \in \Omega_{i}^{-}:=\left\{j \in I \mid E_{j}-T(\lambda(i), \lambda(j))\right.$ $<E_{i}$ with $\lambda(j) \neq \lambda(i)$, and $E_{j}-T(\lambda(i), \lambda(j)) \notin \tau(h)$ for all $h \in I$ such that $\lambda(h)=\lambda(i)\}$. For each $i \in l$, a shipment $Z_{p i}$ that arrives at location $\lambda(i)$ at the beginning $E_{i}$ of a work interval $i$ comes from a location $p \in \Omega_{i}^{+}:=$ $\left\{p \in \Lambda \mid p \neq \lambda(i)\right.$ and $E_{i}-T(p, \lambda(i)) \notin \tau(h)$ for all $h \in I$ such that $\lambda(h)=p\}$. For each $s \in \Lambda$, a shipment $Z_{s j}$ that departs from location $s$ after the final work interval associated with location $s$ arrives at location $\lambda(j)$ at the beginning $E_{j}$ of a work interval $j \in \Psi_{s}:=\left\{j \in l \mid \lambda(j) \neq s, E_{j}-T(s, \lambda(j)) \geq \max \left\{L_{i} \mid \lambda(i)=\right.\right.$ $s\}\}$. If there are no work intervals associated with $s$ ( $s$ is, e.g., a store of machines), then $\Psi_{s}=I$. For each $i \in l$, a shipment $Y_{i j}$ that departs from $\lambda(i)$ at a time instant within a work interval $i$ arrives at location $\lambda(j)$ at a time instant within a work interval $j \in \Phi_{i}^{+}:=\{j \in I \mid \tau(i, j) \neq \varnothing\}$. For each $i \in l$, a shipment $Y_{j i}$ that arrives at $\lambda(i)$ at a time instant within a work interval $i$ comes from location $\lambda(j)$ with a departure time within a work interval $j \in \Phi_{i}{ }^{-}:=\{j \in I \mid \tau(j, i) \neq \varnothing\}$.

In order to reformulate constraints (3) on the availability of machines at the beginning of each work interval $i \in I$, we denote by $J_{i}$ the set of work intervals $j$ preceding the work interval $i$ at the same location $\lambda(i)$, i.e., $J_{i}:=\left\{j \in I \mid \lambda(j)=\lambda(i), E_{j}<E_{i}\right\}$. With the foregoing notation, we formulate Problem 2 as follows:

$$
\text { Minimize } \sum_{i \in l} \sum_{p \in \Omega_{i}^{+}} C(p, \lambda(i)) Z_{p i}+\sum_{i \in l} \sum_{\substack{j \in l \\ j \in i, j \\ \tau(i, j) \neq \varnothing}} C(\lambda(i), \lambda(j)) Y_{i j}
$$

Subject to

$$
\begin{gathered}
0 \leq \sum_{j \in \Omega_{i}^{-}} Z_{\lambda(i) j}+\sum_{h \in J_{i}}\left[-\sum_{p \in \Omega_{h}^{+}} Z_{p h}+\sum_{j \in \Phi_{h}^{+}} Y_{h j}-\sum_{j \in \Phi_{\bar{h}}^{-}} Y_{j h}\right] \\
-\sum_{p \in \Omega_{i}^{+}} Z_{p i}+\sum_{j \in \Phi_{i}^{+}} Y_{i j}-\sum_{j \in \Phi_{i}^{-}} Y_{j i} \leq Q_{\lambda(i)}, \quad \forall i \in l ;
\end{gathered}
$$

$$
\begin{gathered}
0 \leq \sum_{j \in \Omega_{i}^{-}} Z_{\lambda(i) j}+\sum_{h \in J_{i}}\left[-\sum_{p \in \Omega_{h}^{+}} Z_{p h}+\sum_{j \in \Phi_{h}^{+}} Y_{h j}-\sum_{j \in \Phi_{h}^{-}} Y_{j h}\right] \\
-\sum_{p \in \Omega_{i}^{+}} Z_{p i}+\sum_{j \in \Phi_{i}^{+}} Y_{i j}-\sum_{j \in \Phi_{i}^{-}} Y_{j i} \leq Q_{\lambda(i)}, \quad \forall i \in l ; \\
-\sum_{i \in \epsilon_{k}}\left\{\left(L_{i}-E_{i}\right)\left[\sum_{j \in \Omega_{i}^{-}} Z_{\lambda(i) j}+\sum_{h \in J_{i}}\left(-\sum_{p \in \Omega_{h}^{+}} Z_{p h}+\sum_{j \in \Phi_{h}^{+}} Y_{h j}-\sum_{j \in \Phi_{h}^{-}} Y_{j h}\right)-\sum_{p \in \Omega_{i}^{+}} Z_{p i}\right]\right. \\
-\sum_{j \in \Phi_{i}^{+}}\left(L_{i}-t_{i j}\right) Y_{i j}+\sum_{j \in \Phi_{i}^{-}}\left(L_{i}-t_{j i}-T(\lambda(j), \lambda(i)) Y_{j i}\right\} \\
\geq w_{k}-Q_{\lambda^{\prime}(k)} \sum_{i \in l_{k}}\left(L_{i}-E_{i}\right), \quad \forall k \in K ; \\
t_{i j}+T(\lambda(i), \lambda(j)) \in \tau(i, j) \quad \text { for all } i, j \in l \text { such that } \tau(i, j) \neq \\
\varnothing ; \\
Z_{p j} \geq 0, p \in \Lambda, j \in l ; \\
Y_{i j} \geq 0, i, j \in I, \tau(i, j) \neq \varnothing ; \\
Z_{p j} \text { integer, } p \in \Lambda, j \in l ; \\
Y_{i j} \text { integer, } i, j \in l, \quad \tau(i, j) \neq \varnothing ;
\end{gathered}
$$

In addition, condition (iv) must be satisfied for all $i \in I$.

In this problem, we have to find $Z_{p j}, Y_{i j}$ and $t_{i j}$ to minimize the total transportation cost such that constraints (10)-(17) and condition (iv) are satisfied. Unlike constraints (3) of Problem 1, constraints (10) and (11) of Problem 2 represent the restrictions on the machines availability at each location only at the beginning and at the end of each work interval, respectively. Constraints (12) establish that each task $k \in K$ must be accomplished within its work intervals $i \in I_{k}$. Constraints (13) stipulate that each shipment $Y_{i j}$ must arrive at $\lambda(j)$ at time $t_{i j}+T(\lambda(i), \lambda(j))$ within the corresponding projection $\tau(i, j)$. Constraints (14) and (15) impose nonnegativity conditions on the number of machines $Z_{p j}$ transported in class $A$ shipments and on the number of machines $Y_{i j}$ transported in class $B$ shipments, respectively. Finally, constraints (16) and (17) impose integrality conditions on the variables $Z_{p j}$ and $Y_{i j}$, respectively, while condition (iv) ensures that the availability of 
machines is not violated at any time in the interior of each work interval.

Contrary to Problem 1, Problem 2 has a definite number of decision variables. However, this problem is still nonlinear due to the nonlinear terms $\left(L_{i}-t_{i j}\right) Y_{i j}$ and $\left[L_{i}-t_{j i}-T(\lambda(j), \lambda(i))\right] Y_{j i}$ in Equation 12 , and it cannot be solved directly because its constraints on the machines availability in the interior of the work intervals (condition (iv)) cannot be expressed in terms of the decision variables $Z_{p j}$, $Y_{i j}$ and $t_{i j}$. In order to solve Problem 2, we will apply an iterative procedure in which the relaxed Problem 2 (called Problem 2'), obtained by dropping condition (iv) from the set of constraints, is solved iteratively by dividing the work intervals into adjacent subintervals until the solution satisfies such condition.

\section{Problem 3: A mixed-integer linear program equivalent to Problem 2}

In this section, we develop the last stage of the formulation of our model (Problem 3). Since each $\tau(i, j)$ in Equation 13 (and therefore each $\left.\tau^{\prime}(i, j)\right)$ is a bounded convex polyhedron, Problem 2' can be seen as a generalized linear program (see e.g., Dantzig, 1963, and Lasdon, 2002) which may be transformed into a linear program by the same device used in the well-known Dantzig-Wolfe decomposition principle. From this, we can formulate a mixed-integer linear program equivalent to Problem 2'. We call this new program Problem 3.

In the following theorem, we prove that each single class $B$ shipment, which departs from a location $\lambda(i)$ at an instant $t_{i j}$ in the interior of $\tau^{\prime}(i, j)$, is equivalent (in the sense of the portions of workcontent processed by the machines transported in the shipment) to a pair of "extreme shipments", each of them with a departure time equals to an extreme of the time interval $\tau^{\prime}(i, j)$. By the convexity of $\tau^{\prime}(i, j)$, any time instant $t_{i j} \in \tau^{\prime}(i, j)$ can be written as $t_{i j}=\alpha_{i j}(1-\mu)+\beta_{i j} \mu$, with $0<\mu<1$, where $\alpha_{i j}$ is the left-hand extreme of the time interval $\tau^{\prime}(i, j)$ and $\beta_{i j}$ is the right-hand extreme of this time interval. Figure 3 illustrates the extreme shipments $Y_{i j}-x_{i j}$ and $x_{i j}$ and the corresponding consolidated shipment $Y_{i j}$.

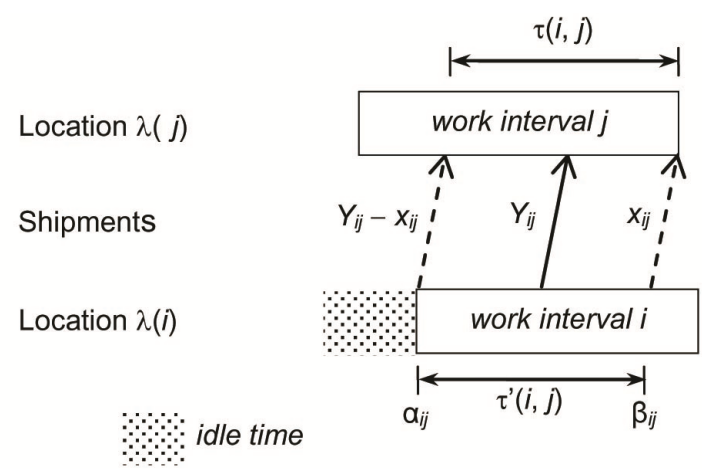

Figure 3. Extreme shipments $Y_{i j}-x_{i j}$ and $x_{i j}$.

Theorem 3. Let $i, j \in I$ be two work intervals such that $\lambda(i) \neq \lambda(j)$ and $\tau^{\prime}(i, j) \neq \varnothing$. Suppose that a single shipment of $Y_{i j}$ machines departs from location $\lambda(i)$ at time $t_{i j} \in \tau^{\prime}(i, j)$ and arrives at location $\lambda(j)$ at time $t_{i j}+T(\lambda(i), \lambda(j)) \in \tau(i, j)$. Then, both the work-content processed by the $Y_{i j}$ machines at location $\lambda(i)$ during work interval $i$ and the work-content processed by the same $Y_{i j}$ machines at location $\lambda(j)$ during work interval $j$ remain unchanged if the single shipment of $Y_{i j}$ machines is replaced by the following two extreme shipments of $x_{i j}$ and $Y_{i j}-x_{i j}$ (not necessarily integers) machines, respectively:

(a) Right-extreme shipment: a shipment of $x_{i j}:=Y_{i j}$ $\left(t_{i j}-\alpha_{i j}\right) /\left(\beta_{i j}-\alpha_{i j}\right)$ machines departs from location $\lambda(i)$ at the end of the time interval $\tau^{\prime}(i, j), \beta_{i j}$, and arrives at location $\lambda(j)$ at the end of the time interval $\tau(i, j)$;

(b) Left-extreme shipment: a shipment of $Y_{i j}-x_{i j}$ machines departs from location $\lambda(i)$ at the beginning of the time interval $\tau^{\prime}(i, j), \alpha_{i j}$, and arrives at location $\lambda(j)$ at the beginning of the time interval $\tau(i, j)$.

\section{Proof}

By using Equation 1, the sum of the work-content processed by the $x_{i j}$ machines at location $\lambda(j)$ during work interval $j$ and the work-content processed by the $Y_{i j}-x_{i j}$ machines at location $\lambda(j)$ during work interval $j$ is given by

$x_{i j} G\left(j, \beta_{i j}+T(\lambda(i), \lambda(j))\right)+\left(Y_{i j}-x_{i j}\right) G\left(j, \alpha_{i j}+T(\lambda(i)\right.$, $\lambda(j)))=$

$=x_{i j}\left(L_{j}-\beta_{i j}-T(\lambda(i), \lambda(j))\right)+\left(Y_{i j}-x_{i j}\right)\left(L_{j}-\alpha_{i j}-T\right.$ $(\lambda(i), \lambda(j))$ 
$=x_{i j}\left(\left(L_{j}-\beta_{i j}-T(\lambda(i), \lambda(j))-\left(L_{j}-\alpha_{i j}-T(\lambda(i), \lambda(j))\right)+\right.\right.$ $+Y_{i j}\left(L_{j}-\alpha_{i j}-T(\lambda(i), \lambda(j))=x_{i j}\left(\alpha_{i j}-\beta_{i}\right)+Y_{i j}\left(L_{j}-\alpha_{i j}-\right.\right.$ $T(\lambda(i), \lambda(j))$.

Replacing $x_{i j}$ by $Y_{i j}\left(t_{i j}-\alpha_{i j}\right) /\left(\beta_{i j}-\alpha_{i j}\right)$ in the last equality, we obtain

$\left(\alpha_{i j}-\beta_{i}\right) Y_{i j}\left(t_{i j}-\alpha_{i j}\right) /\left(\beta_{i j}-\alpha_{i j}\right)+Y_{i j}\left(L_{j}-\alpha_{i j}-T(\lambda(i)\right.$, $\lambda(j))=$

$=Y_{i j}\left(L_{j}-\alpha_{i j}-T(\lambda(i), \lambda(j))-Y_{i j}\left(t_{i j}-\alpha_{i j}\right)\right.$

$=Y_{i j}\left(L_{j}-T(\lambda(i), \lambda(j))-Y_{i j}\left(t_{i j}\right)\right.$

$=Y_{i j}\left(L_{j}-t_{i j}-T(\lambda(i), \lambda(j))\right)=Y_{i j} G\left(j, t_{i j}+T(\lambda(i), \lambda(j))\right)$,

where $Y_{i j} G\left(j, t_{i j}+T(\lambda(i), \lambda(j))\right)$ is the work-content that $Y_{i j}$ machines, after arriving at location $\lambda(j)$ at time $t_{i j}+T(\lambda(i), \lambda(j))$, process during work interval $j$.

The proof is similar for work interval $i$.

Notice that from the definition of $x_{i j}$, we have

$$
t_{i j}=\alpha_{i j}+\left(\beta_{i j}-\alpha_{i j}\right) x_{i j} / Y_{i j}, \quad Y_{i j}>0
$$

and hence the parameter $\mu$ in $t_{i j}=\alpha_{i j}(1-\mu)+\beta_{i j} \mu$ is equal to $x_{i j} / Y_{i j}$.

Theorem 3 expresses that each consolidated shipment of $Y_{i j}$ machines departing from $\lambda(i)$ at a time instant $t_{i j} \in \tau^{\prime}(i, j)$ is equivalent to two extreme shipments of $x_{i j}$ and $Y_{i j}-x_{i j}$ machines, respectively. This equivalence allows us to transform Problem 2' into a linear problem, as shown in the following corollary.

\section{Corollary of Theorem 3}

(a) Both the pair of variables $\left(Y_{i j}, t_{i j}\right)$ describing a single consolidated shipment and the pair of variables $\left(Y_{i j}, X_{i j}\right)$ describing the corresponding pair of extreme shipments are related by $x_{i j}=\left(t_{i j}-\alpha_{i j}\right) Y_{i j}$ I $\left(\beta_{i j}-\alpha_{i j}\right)$ or, equivalently, by Equation 18;

(b) $0 \leq x_{i j} \leq Y_{i j}$;

(c) Problem 2', which is formulated in terms of variables $\left(Z_{p j}, Y_{i j}, t_{i j}\right)$, can be transformed by using Equation 18 in a linear program in terms of variables $\left(Z_{p j}, Y_{i j}, X_{i j}\right)$. Specifically, the set of constraints (12), which contains all the nonlinearities of Problem 2', can be transformed into the following set of linear constraints:

$$
\begin{aligned}
& -\sum_{i \in \in_{k}}\left\{\left(L_{i}-E_{i}\right)\left[\sum_{j \in \Omega_{i}^{-}} Z_{\lambda(i) j}+\sum_{h \in J_{i}}\left(-\sum_{p \in \Omega_{h}^{+}} Z_{p h}+\sum_{j \in \Phi_{h}^{+}} Y_{h j}-\sum_{j \in \Phi_{h}^{-}} Y_{j h}\right)-\sum_{p \in \Omega_{i}^{+}} Z_{p i}\right]\right. \\
& -\sum_{j \in \Phi_{i}^{+}}\left[\left(L_{i}-\alpha_{i j}\right) Y_{i j}-\left(\beta_{i j}-\alpha_{i j}\right) x_{i j}\right]+\sum_{j \in \Phi_{i}^{-}}\left[\left(L_{i}-\alpha_{j i}-T(\lambda(j), \lambda(i)) Y_{j i}-\left(\beta_{j i}-\alpha_{j i}\right) x_{j i}\right]\right\} \\
& \geq w_{k}-Q_{\lambda^{\prime}(k)} \sum_{i \in \epsilon_{k}}\left(L_{i}-E_{i}\right), \quad \forall k \in K .
\end{aligned}
$$

Proof

(a) Immediate from Theorem 3.

(b) This part is immediate from that $Y_{i j}>0$.

(c) Substituting Equation 18 in the nonlinear terms of Equation 12, given by $\left(L_{i}-t_{i j}\right) Y_{i j}$ and $\left[L_{i}-t_{j i}-\right.$ $T(\lambda(j), \lambda(i))] Y_{j i}$, and making simplifications, we have

$\left(L_{i}-t_{i j}\right) Y_{i j}=\left(L_{i}-\left(\alpha_{i j}+\left(\beta_{i j}-\alpha_{i j}\right) x_{i j} / Y_{i j}\right)\right) Y_{i j}$

$=\left(L_{i} Y_{i j}-\left(\alpha_{i j} Y_{i j}+\left(\beta_{i j}-\alpha_{i j}\right) x_{i j}\right)\right)$

$=\left(L_{i}-\alpha_{i j}\right) Y_{i j}-\left(\beta_{i j}-\alpha_{i j}\right) X_{i j}$;

$\left[L_{i}-t_{j i}-T(\lambda(j), \lambda(i))\right] Y_{j i}=\left[L_{i}-\left(\alpha_{j i}+\left(\beta_{j i}-\alpha_{j i}\right) x_{j i} / Y_{j i}\right)\right.$

$-T(\lambda(j), \lambda(i))] Y_{j i}$

$=L_{i} Y_{j i}-\alpha_{j i} Y_{j i}-\left(\beta_{j i}-\alpha_{j i}\right) x_{j i}-T(\lambda(j), \lambda(i)) Y_{j i}$

$=L_{i} Y_{j i}-\alpha_{j i} Y_{j i}-T(\lambda(j), \lambda(i)) Y_{j i}-\left(\beta_{j i}-\alpha_{j i}\right) x_{j i}$

$=\left(L_{i}-\alpha_{j i}-T(\lambda(j), \lambda(i))\right) Y_{j i}-\left(\beta_{j i}-\alpha_{j i}\right) x_{j i}$.

We formulate Problem 3 as the program that consists of finding $Z_{p j}, Y_{i j}$ and $x_{i j}$ to minimize the total transportation cost (9) such that constraints (10), (11), (14), (16), (17), (19), $Y_{i j} \geq x_{i j}$, and $x_{i j} \geq 0$ are satisfied. As one can see, Problem 3 is a mixed-integer linear program.

It is worth notice that an optimal solution $\left\{Z_{p j}, Y_{i j}\right.$, $\left.x_{i j}\right\}$ of Problem 3 in which the shipments $x_{i j}$ and $Y_{i j}-$ $x_{i j}$ depart respectively from an extreme of the time interval $\tau^{\prime}(i, j)$ determines, through Equation 18, a unique optimal solution $\left\{Z_{p j}, Y_{i j}, t_{i j}\right\}$ of Problem 2' in which the departure time $t_{i j}$ of the consolidated shipment $Y_{i j}$ (integer) is a time instant in the interior of $\tau^{\prime}(i, j)$. Reciprocally, an optimal solution $\left\{Z_{p j}, Y_{i j}\right.$, $\left.t_{i j}\right\}$ of Problem 2' determines, through (a) and (b) of Theorem 3 , a unique optimal solution $\left\{Z_{p j}, Y_{i j}, x_{i j}\right\}$ of Problem 3.

A solution $\left\{Z_{p j}, Y_{i j}, t_{i j}\right\}$ of Problem 2' solves Problem 2 (and then Problem 1 ) only if such solution satisfies condition (iv) for all $i \in l$. If an optimal solution $\left\{Z_{p j}, Y_{i j}, t_{i j}\right\}$ does not satisfy condition (iv), then the following relaxation procedure can be used to solve Problem 2. 


\subsection{A relaxation procedure to solve Problem 2}

We propose a relaxation procedure to solve Problem 2. This procedure consists of iteratively solving the linearized Problem 2' (Problem 3) and takes advantage of the fact that constraints (10) and (11) respectively ensure that the machines availability at the beginning and at the end of each work interval is not violated. Our procedure is based on the following two observations: (a) If the work intervals are arbitrarily divided into adjacent subintervals, then any optimal solution of Problem 2 with the divided work intervals is also an optimal solution of the original Problem 2; (b) If, in particular, each work interval is divided such that there is at most one class $B$ shipment in the interior of each subinterval, then constraints (10) and (11) ensure also that condition (iv) is satisfied for every subinterval. Next we propose a strategy of dividing the work intervals that is computationally more efficient than the one just described in (b).

If a solution $\left\{Z_{p j}, Y_{i j}, x_{i j}\right\}$ of Problem 3 does not satisfy condition (iv), i.e., the corresponding solution $\left\{Z_{p j}, Y_{i j}, t_{i j}\right\}$ does not solve Problem 2, then at each location, the work interval whose interior contains the earliest time at which condition (iv) was violated is divided into two adjacent work intervals separated by that earliest time. Then Problem 3 with the divided work intervals is solved, and so on until this condition on the availability of machines is not violated for all the work intervals. This procedure converges because the number of shipments of machines and the number of work intervals are finite. Formally, this procedure is as follows:

Step 1. Solve Problem 3 and denote by $\left\{Z_{p j}, Y_{i j}, x_{i j}\right\}$ the corresponding optimal solution. Use Equation 18 to find the corresponding solution $\left\{Z_{p j}, Y_{i j}, t_{i j}\right\}$.

Step 2. For each $p \in \Lambda$, let $\theta_{p}$ be the set of all instants $t_{i j}$ within the work intervals associated with location $p=\lambda(i)$ such that condition (iv) is violated, i.e., $\theta_{p}:=\left\{t_{i j} \mid \lambda(i)=p, t_{i j} \in \tau^{\prime}(i, j) \neq \varnothing\right.$, and condition (iv) is violated for location $p$ at time $\left.t_{i j}\right\}$. For each $p \in \Lambda$, let $v_{p}:=\min \theta_{p}$ if $\theta_{p} \neq \varnothing$ and let $v_{p}:=\infty$ if $\theta_{p}=$ $\varnothing$. If $v_{p}=\infty$ for all $p \in \Lambda$ then $\left\{Z_{p j}, Y_{i j}, t_{i j}\right\}$ is a solution of Problem 2, and therefore of Problem 1; in this case, the procedure finishes. If $v_{p} \neq \infty$ for some $p \in \Lambda$, then go to Step 3 .

Step 3. For each $p \in \Lambda$ such that $v_{p} \neq \infty$, divide the work interval containing the instant $v_{p}$ into two adjacent work intervals separated by the time $v_{p}$; reformulate Problem 3 considering the divided work intervals. Return to Step 1.

\subsection{Problem 3 in terms of matrices}

Problem 3 can be written in matrix form. Let $\mathbf{y}$ be the vector whose components are $Z_{i j}$ and $Y_{i j}$, and let $\mathbf{x}$ be the vector whose components are $x_{i j}$. Then, Problem 3 can be expressed as follows:

Mininimize Cy

Subject to

$A_{1} \mathbf{x}+A_{2} \mathbf{y} \geq \mathbf{b}_{1}$

$A_{3} \mathbf{y} \geq \mathbf{b}_{2}$

$\mathbf{x}, \mathbf{y} \geq \mathbf{0}$

$\mathbf{y}$ integer.

The objective function (20) minimizes the total transportation cost. Constraints (21) correspond to constraints (19) and guarantee that each task $k \in K$ is accomplished within its prescribed work intervals. Each element of $A_{1}$ is either a term of the form $-\left(\beta_{i j}-\alpha_{i j}\right)$ or a term of the form $\left(\beta_{j i}-\alpha_{j i}\right)$; these terms correspond to the coefficients of the shipments $x_{i j}$ and $x_{j i}$, respectively. Similarly, each element of $A_{2}$ is either a term of the form $L_{i}-\alpha_{i j}$ or a term of the form $-\left(L_{i}-\alpha_{j i}-T(\lambda(j), \lambda(i))\right)$; these terms correspond to the coefficients of the shipments $Y_{i j}$ and $Y_{j i}$, respectively. Each element of $\mathbf{b}_{1}$ is of the form $w_{k}-Q_{\lambda^{\prime}(k)} \Sigma_{i}\left(L_{i}-E_{i}\right)$. Constraints (22) contain constraints (10) and (11), and they represent the availability condition of machines at each location at the extremes of each work interval. Each element of $A_{3}$ is 1,0 , or - 1. Each element of $\mathbf{b}_{2}$ is a term of the form $-Q_{\lambda(i)}$. Constraints (23) impose nonnegativity conditions on the variables $Z_{i j}, Y_{i j}$ and $x_{i j}$. Constraints (24) impose integrality conditions on the variables $Z_{i j}$ and $Y_{i j}$. Due to the structure of Problem 3, it can be solved by the method of Benders decomposition.

Finally, we want to remark that Problem 3 is 
equivalent to Problem 2', and that any optimal solution of Problem 2' that satisfies condition (iv) is also an optimal solution of Problem 2 and therefore of Problem 1.

\section{Conclusions}

In this paper, we have developed a new model to obtain an optimal transportation schedule of identical mobile machines which have to execute tasks located at several locations. Our work, motivated by a transportation scheduling problem faced by road construction companies, may be applied in a wide variety of situations. In this problem, the machines can be transported from one location to another location at any time; thus the problem has an indefinite number of variables. However, this indefinite number of variables can be reduced to a definite one because the problem has an optimal solution in which the arrival times of the machines occur only at certain time instants. The constraints ensuring that each task is accomplished within its prescribed time intervals have products of two quantities: the number of machines arriving at a location during a time interval and the amount of time that these machines work during that time interval. These two quantities are decision variables, and hence the problem is nonlinear. Such nonlinearities are avoided by taking advantage of the fact that, at each location, the arrival times of the machines form intervals which are bounded convex polyhedra. Then, we conclude that our problem is indeed a generalized linear program which can be transformed into a mixed-integer linear program by the same device used in the decomposition principle of Dantzig-Wolfe.

\section{References}

[1] Averbakh, I., \& Berman, O., A simple heuristic for m-machine flow-shop and its applications in routing-scheduling problems, Operations Research, Vol. 47, No. 1, 1999, pp. 165-170.

[2] Averbakh, I., Berman, O., \& Chernykh, I., The routing open-shop problem on a network: Complexity and approximation. European Journal of Operational Research, Vol. 173, 2006, pp. 531539.

[3] Bredström, D., \& Rönnqvist, M., Combined vehicle routing and scheduling with temporal precedence and synchronization constraints, European Journal of Operational Research, Vol. 191, 2008, pp. 19-31.

[4] Kek, A. G. H., Cheu, R. L., \& Meng, Q., Distance-constrained capacitated vehicle routing problems with flexible assignment of start and end depots, Mathematical and Computer Modelling, Vol. 47, 2008, pp. 140-152.

[5] Kim, B. I., Kim, S., \& Sahoo, S., Waste collection vehicle routing problem with time windows, Computers and Operations Research, Vol. 33, 2006, pp. 3624-3642.

[6] Desaulniers, G., Lavigne, J., \& Soumis, F., Multi-depot vehicle scheduling problems with time windows and waiting costs, European Journal of Operational Research, Vol. 111, 1998, pp. 479494.

[7] Desrosiers, J., Dumas, Y., Solomon, M., \& Soumis, F., Time constrained routing and scheduling, in Handbooks in operations research and management science. Vol. 8, Network routing, M. O. Ball, T.L. Magnanti, C. L. Monma, \& G. L. Nemhauser Editors, Amsterdam: Elsevier, 1995, pp. 35-139.

[8] Dantzig, G. B., Linear programming and extensions. New York: Princeton Univ. Press, 1963.

[9] Lasdon, L. S., Optimization theory for large systems. New York: Dover Publications Inc. 2002. 